Pacific Journal of Mathematic 


\title{
ON GENERALIZATIONS OF ALTERNATIVE ALGEBRAS
}

\author{
JOYCE LONGMAN
}

Let $A$ be a noncommutative Jordan algebra in which $([x, y], z, z)=0$ for all $x, y, z$ in $A$. In this paper the result of Block [4] and Shestakov [13] that a simple finite dimensional such algebra over a field of characteristic $\neq 2$ is either alternative or Jordan is extended to the infinite dimensional case with idempotent. In the case of a noncommutative Jordan algebra satisfying the weaker identity $([x, y], y, y)=0$ for all $x, y$.in the algebra, a simple finite dimensional such algebra is shown to be commutative, alternative, or an algebra of degree two.

In $\$ 2$ we consider in the first case, power associative rings which satisfy $\left(w, x^{2}, z\right)=x \cdot(w, x, z)$ and $([x, y], y, y)=$ 0 , and in the second case, flexible rings satisfying $\left(w, x^{2}, z\right)=$ $x \cdot(w, x, z)+(x, x,[w, z])$. Under certain conditions the rings are shown to be noncommutative Jordan or alternative respectively.

Throughout this paper all algebras considered are assumed to be algebras over a field of characteristic not two and all rings are assumed to be 2-torsion free (i.e., if $2 a=0$ for $a$ in $R$ then $a=0$ ).

1. Nearly alternative algebras. Let $A$ be a nonassociative algebra. As is usual for $x, y, z$ in $A$ we denote the associator $(x y) z-x(y z)$ by $(x, y, z)$ and the commutator $x y-y x$ by $[x, y] . A$ is flexible if $(x, y, x)=0$, alternative if $(x, x, y)=(y, x, x)=0$, and noncommutative Jordan if $(x, y, x)=\left(x^{2}, y, x\right)=0$.

An algebra $A$ is called simple if $A$ is not a zero algebra, and the only ideals of $A$ are the zero ideal and $A$ itself. In case $A_{K}=$ $A \bigotimes_{F} K$ is simple for every extension $K \supseteqq F$ then $A$ over $F$ is called central simple.

We shall call a noncommutative Jordan algebra $A$ nearly alternative if $A$ satisfies the following identity for all $x, y, z$ in $A$ :

$$
([x, y], z, z)=0 \text {. }
$$

Shestakov [13] called such an algebra "almost alternative." However we choose not to use that terminology since Albert [2] had previously called other algebras by the name "almost alternative."

THEOREM 1.1. If $A$ is a simple nearly alternative algebra with an idempotent $e \neq 1$ then $A$ is commutative or alternative. 
Proof. It is shown by Shestakov [13] that if $A$ is a noncommutative Jordan algebra with idempotent $e \neq 1$ satisfying $([x, y], z, z)=$ 0 then $A$ has the following Peirce decomposition:

$$
A=A_{1}+A_{10}+A_{1 / 21 / 2}+A_{01}+A_{0},
$$

where

$$
A_{i}=\{x \in A \mid e x=x e=i x\}, i=0,1
$$

and

$$
A_{i j}=\{x \in A \mid e x=i x, x e=j x\}, i+j=1, i, j=0, \frac{1}{2}, 1 .
$$

Shestakov also showed that multiplication of elements of the different components is given in the following chart:

\begin{tabular}{r|cclll}
\multicolumn{1}{l}{} & $A_{1}$ & $A_{10}$ & $A_{1 / 21 / 2}$ & $A_{01}$ & $A_{0}$ \\
\cline { 2 - 6 }$A_{1}$ & $A_{1}$ & $A_{10}$ & $A_{1 / 21 / 2}$ & 0 & 0 \\
$A_{10}$ & 0 & $A_{01}$ & $A_{01}$ & $A_{1}$ & $A_{10}$ \\
$A_{1 / 2} 1 / 2$ & $A_{1 / 21 / 2}$ & $A_{01}$ & $A_{1}+A_{10}+A_{01}+A_{0}$ & $A_{10}$ & $A_{1 / 21 / 2}$ \\
$A_{01}$ & $A_{01}$ & $A_{0}$ & $A_{10}$ & $A_{10}$ & 0 \\
$A_{0}$ & 0 & 0 & $A_{1 / 21 / 2}$ & $A_{01}$ & $A_{0}$
\end{tabular}

that $B=A_{10}+A_{01}+A_{10} A_{10}+A_{01} A_{10}$ is an ideal of $A$, and that $x y=$ $-y x$ for any $x, y$ in $A_{i j}(i \neq j)$. Furthermore, if $A_{10}=A_{01}=0$ then $x y=y x$ for all $x, y$ in $A_{1 / 2} 1 / 2$.

Before proceeding to the proof of the theorem, we note the following:

LEMMA 1.1. If $A_{1 / 21 / 2}=0$ then $A$ is alternative.

Proof. Since $A$ is simple, the ideal $B=0$ or $B=A$. If $B=0$, then $A_{10}=A_{01}=0$ and $A=A_{1}+A_{0}$. This implies $e=1$, a contradiction. Hence $B=A$, and $A_{1}=A_{10} A_{01}, A_{0}=A_{01} A_{10}$. We prove $A$ is alternative by showing

$$
(x, y, z)=\varepsilon(\sigma)(\sigma(x), \sigma(y), \sigma(z))
$$

for all permutations $\sigma$, with $\varepsilon(\sigma)=1$ or -1 respectively for $\sigma$ even or odd. It suffices to show that (1.2) holds for all possible choices of $x, y, z$ in the component subspaces. Since $A$ is noncommutative Jordan, it has been shown by Florey [5] that $A$ satisfies the identity

$$
\left(w, x^{2}, z\right)=x \cdot(w, x, z)
$$


for all $x, w, z$ in $A$ where $x \cdot y=x y+y x$. A linearization of (1.3) yields

$$
(w, x \cdot y, z)=x \cdot(w, y, z)+y \cdot(w, x, z) .
$$

Now suppose $x_{1}, y_{1}, z_{1} \in A_{1}$. Since $y_{1}=w_{10} w_{01}$,

$$
\left(x_{1}, y_{1}, z_{1}\right)=\left(x_{1}, w_{10} \cdot w_{01}, z_{1}\right)=w_{10} \cdot\left(x_{1}, w_{01}, z_{1}\right)+w_{01} \cdot\left(x_{1}, w_{10}, z_{1}\right)=0 \text {. }
$$

Hence $\left(A_{1}, A_{1}, A_{1}\right)$ alternates. We show that the remaining thirty six associators with $A_{1}$ in any position alternate.

By the Peirce multiplication chart and flexibility,

$$
\begin{aligned}
\left(A_{1}, A_{1}, A_{01}\right) & =\left(A_{01}, A_{1}, A_{1}\right)=\left(A_{1}, A_{01}, A_{1}\right)=\left(A_{1}, A_{0}, A_{1}\right)=\left(A_{1}, A_{0}, A_{0}\right) \\
& =\left(A_{0}, A_{0}, A_{1}\right)=\left(A_{0}, A_{1}, A_{0}\right)=\left(A_{0}, A_{1}, A_{1}\right)=\left(A_{1}, A_{1}, A_{0}\right) \\
& =0
\end{aligned}
$$

Again from flexibility and the multiplication chart each of the associators $\left(A_{1}, A_{1}, A_{10}\right),\left(A_{1}, A_{01}, A_{10}\right),\left(A_{1}, A_{0}, A_{10}\right)$, and $\left(A_{0}, A_{1}, A_{01}\right)$ alternates.

Now suppose $x_{1} \in A_{1}, y_{10}, z_{10} \in A_{10}$. Linearizing (1.1) and the flexible law $(x, y, x)=0$, we obtain

$$
([x, y], z, w)+([x, y], w, z)=0
$$

and

$$
(x, y, z)+(z, y, x)=0
$$

Then

$$
\begin{aligned}
\left(x_{1}, y_{10}, z_{10}\right) & =\left(x_{1}, y_{10},\left[e, z_{10}\right]\right)-\left(y_{10}, x_{1}, z_{10}\right)=\left(z_{10}, x_{1}, y_{10}\right) \\
& =\left(\left[e, z_{10}\right], x_{1}, y_{10}\right)=-\left(z_{10}, y_{10}, x_{1}\right) .
\end{aligned}
$$

Also $-\left(z_{10}, y_{10}, x_{1}\right)=-\left(y_{10}, x_{1}, z_{10}\right)=-\left(\left[e, y_{10}\right], x_{1}, z_{10}\right)=\left(y_{10}, z_{10}, x_{1}\right)=$ $-\left(x_{1}, z_{10}, y_{10}\right)$ by (1.5) and (1.6). This shows $\left(A_{1}, A_{10}, A_{10}\right)$ alternates. In the same manner $\left(A_{1}, A_{01}, A_{01}\right)$ alternates. Therefore every associator with $A_{1}$ or in an analogous manner with $A_{0}$ in any position alternates.

We have reduced the proof to the case in which $x, y, z \in A_{i j}+$ $A_{j i}, i, j=0,1, i+j=1$. Again using (1.5) and (1.6),

$$
\begin{aligned}
\left(x_{i j}, y_{i j}, z_{i j}\right) & =i\left(x_{i j}, y_{i j},\left[e, z_{i j}\right]\right)-j\left(x_{i j}, y_{i j},\left[e, z_{i j}\right]\right) \\
& =-i\left(y_{i j}, x_{i j},\left[e, z_{i j}\right]\right)+j\left(y_{i j}, x_{i j},\left[e, z_{i j}\right]\right) \\
& =i\left(\left[e, z_{i j}\right], x_{i j}, y_{i j}\right)-j\left(\left[e, z_{i j}\right], x_{i j}, y_{i j}\right) \\
& =-i\left(x_{i j}, z_{i j},\left[e, y_{i j}\right]\right)+j\left(x_{i j}, z_{i j},\left[e, y_{i j}\right]\right) \\
& =-\left(x_{i j}, z_{i j}, y_{i j}\right)=\left(y_{i j}, z_{i j}, x_{i j}\right) .
\end{aligned}
$$

Also

$$
\begin{aligned}
\left(z_{i j}, x_{i j}, y_{i j}\right) & =i\left(\left[e, z_{i j}\right], x_{i j}, y_{i j}\right)-j\left(\left[e, z_{i j}\right], x_{i j}, y_{i j}\right) \\
& =-i\left(\left[e, z_{i j}\right], y_{i j}, x_{i j}\right)+j\left(\left[e, z_{i j}\right], y_{i j}, x_{i j}\right) \\
& =-\left(z_{i j}, y_{i j}, x_{i j}\right) .
\end{aligned}
$$


Combining these results yields $\left(A_{i j}, A_{i j}, A_{i j}\right)$ alternates. The case $x, y \in A_{i j}$ and $z \in A_{j i}$ is proved in a similar manner. Thus $\left(A_{i j}, A_{i j}, A_{j i}\right)$ alternates and the lemma is proved.

We are now in a position to complete our main result. Assume $A$ is not alternative. By the simplicity of $A$, the ideal $B$ must be $A$ or the zero ideal. We found by Lemma 1.1 that $B=A$ implied $A$ was alternative. Thus we are left with $B=0$ from which it follows that $A_{10}=A_{01}=0$ and $A=A_{1}+A_{1 / 21 / 2}+A_{0}$ We next observe that $\left[x, A_{1 / 21 / 2}\right]=0$ for all $x$ in $A$. For if $x \in A_{i}, i=0,1, y \in A_{1 / 21 / 2}$, then $(x, e, y)=-(y, e, x)$ by flexibility implies $(x e) y-x(e y)=-(y e) x+$ $y(e x)$ so that $x y=y x$. Shestakov [13] has proved $x y=y x$ for $x, y$ in $A_{1 / 21 / 2}$.

Next we show that $x y=y x$ for $x, y \in A_{i}, i=0,1$. McCrimmon [8] has shown that $D=\left(A_{1 / 2} A_{1 / 2}\right)_{0}+A_{1 / 2}+\left(A_{1 / 2} A_{1 / 2}\right)_{1}$ is an ideal of $A$ where $A$ is a noncommutative Jordan algebra and $A=A_{0}+A_{1 / 2}+A_{1}$. In our case $A_{1 / 2}=A_{1 / 2}$. If $D=0$ then $A_{1 / 21 / 2}=0$ and $e=1$, a contradiction. If $D=A$ then $A_{1}=\left(A_{1 / 2} A_{1 / 2}\right)_{1}$ and $A_{0}=\left(A_{1 / 2} A_{1 / 2}\right)_{0}$. Let $x, y \in A_{i}, i=0,1$. Then $x=(u w)_{i}, y=(z t)_{i}$ where $u, w, z, t \in A_{1 / 2}$. In a flexible ring the equation

$$
[x \cdot y, z]=x \cdot[y, z]+[x, z] \cdot y
$$

holds [13]. Thus $2[u w, z t]=[u \cdot w, z t]=u \cdot[w, z t]+[u, z t] \cdot w$. But $z t \in A_{1}+A_{0}$ implies $[w, z t]=0$ and $[u, z t]=0$ since $\left[A_{1 / 2} 1_{1 / 2}, A\right]=0$. Hence $2[u w, z t]=0$ and $x y=y x$ in $A_{i}, i=0,1$. $A$ is therefore commutative, and the theorem is proved.

We next consider a noncommutative Jordan algebra $A$ which satisfies the following identity for all $x, y$ in $A$ :

$$
([x, y], y, y)=0 \text {. }
$$

LEMMA 1.2. If $A$ is a noncommutative Jordan algebra which satisfies (1.8) then the identity

$$
\begin{aligned}
(x \cdot y, z, w) & +(x, y, z \cdot w)=x \cdot(y, z, w)+y \cdot(x, z, w) \\
& +z \cdot(x, y, w)+w \cdot(x, y, z)
\end{aligned}
$$

holds in $A$.

Proof. We use the Teichmüller identity

$$
(x, y z, w)=(x y, z, w)+(x, y, z w)-x(y, z, w)-(x, y, z) w
$$

and flexibility to obtain $(x, y \cdot z, w)=(x, y z, w)+(x, z y, w)=(x, y z, w)-$ $(w, z y, x)=(x y, z, w)+(x, y, z w)-x(y, z, w)-(x, y, z) w-(w z, y, x)-$ $(w, z, y x)+w(z, y, x)+(w, z, y) x=(x \cdot y, z, w)+(x, y, z \cdot w)-w \cdot(x, y, z)-$ 
$x \cdot(y, z, w)$. Next we apply $(1.4)$ to $(x, y, z \cdot w)$ to get $y \cdot(x, z, w)+$ $z \cdot(x, y, w)=(x \cdot y, z, w)+(x, y, z \cdot w)=x \cdot(y, z, w)+y \cdot(x, z, w)+$ $z \cdot(x, y, w)+w \cdot(x, y, z)$ and the lemma is proved.

We now follow a process similar to that of Shestakov [13] to classify a central simple finite dimensional noncommutative Jordan algebra satisfying (1.8).

Theorem 1.2. If $A$ is a simple finite dimensional noncommutative Jordan algebra satisfying identity (1.8) then $A$ is alternative, commutative, or an algebra of degree two.

Proof. By considering $A$ over its centroid and taking a scalar extension, we see that it is enough to prove the theorem when the base field $F$ is algebraically closed. Then by the known classification of central simple noncommutative Jordan algebras [8] $A$ has one of the following forms:

(1) $A$ is a Jordan algebra;

(2) $A$ is a quasiassociative algebra, i.e., $A$ is isomorphic to $B$ as vector spaces, where $B$ is a complete matrix algebra over $F, \lambda \neq$ $1 / 2$ in $F$, with multiplication $(x y)_{A}=(x \cdot y)_{B}+(1-\lambda)(y \cdot x)_{B}$;

(3) $A$ is an algebra of degree one or two.

Assume $A$ is not commutative, i.e., Case 1 does not hold. Suppose Case 2 holds. The identity $([x, y], y, y)=0$ implies

$$
([x, y] y) y-[x, y] y^{2}=0
$$

in $A$. Then

$$
\begin{aligned}
{[x, y]_{A} } & =(x y)_{A}-(y x)_{A}=\lambda x \cdot y+(1-\lambda) y \cdot x-\lambda y \cdot x-(1-\lambda) x \cdot y \\
& =(2 \lambda-1)[x, y]_{B} .
\end{aligned}
$$

We have in $B$,

$$
\begin{gathered}
(2 \lambda-1)\left\{\lambda\left(\lambda[x, y]_{B} \cdot y+(1-\lambda) y \cdot[x, y]_{B}\right) \cdot y+(1-\lambda) y \cdot\left[\lambda[x, y]_{B} \cdot y\right.\right. \\
\left.\left.+(1-\lambda) y \cdot[x, y]_{B}\right]-\lambda[x, y]_{B} \cdot y^{2}-(1-\lambda) y^{2} \cdot[x, y]_{B}\right\}=0 .
\end{gathered}
$$

This yields $(2 \lambda-1)\left[\lambda^{2}[x, y]_{B} \cdot y^{2}+\lambda(1-\lambda) y \cdot[x, y]_{B} \cdot y+(1-\lambda) \lambda y \cdot[x, y]_{B} \cdot y+\right.$ $\left.(1-\lambda)^{2} y^{2} \cdot[x, y]_{B}-\lambda[x, y]_{B} \cdot y^{2}-(1-\lambda) y^{2} \cdot[x, y]_{B}\right]=0$ which becomes $(2 \lambda-1)\left[\lambda(\lambda-1)[x, y]_{B} \cdot y^{2}+(1-\lambda)(-\lambda) y^{2} \cdot[x, y]_{B}+2 \lambda(1-\lambda) y \cdot[x, y]_{B} \cdot y\right]=$ 0 or $(2 \lambda-1) \lambda(\lambda-1)\left[[x, y]_{B} \cdot y^{2}+y^{2} \cdot[x, y]_{B}-2 y \cdot[x, y]_{B} \cdot y\right]=0$. If $\lambda \neq$ 0,1 then $[x, y]_{B} \cdot y^{2}+y^{2} \cdot[x, y]_{B}-2 y \cdot[x, y]_{B} \cdot y=0$. With the elements $x=e_{12}, y=e_{11}, z=e_{22}$ from the usual matrix basis we have $\left[e_{12}, e_{11}\right] \cdot e_{22}^{2}+$ $e_{22}^{2} \cdot\left[e_{12}, e_{11}\right]-2 e_{22} \cdot\left[e_{12}, e_{11}\right] \cdot e_{22}=0$ and $\left(-e_{12}\right) \cdot e_{22}-2 e_{22} \cdot\left(-e_{12}\right) \cdot e_{22}+e_{22} \cdot\left(-e_{12}\right)=$ 0 implies $e_{12}=0$, a contradiction. 
Kleinfeld and Kokoris [6] have shown there are no simple noncommutative Jordan algebras of degree one over a field $F$ of characteristic 0. Kokoris has classified the nodal noncommutative Jordan algebras over a field of characteristic $p \neq 2$ [7]. Block's proof that there are no nearly alternative such algebras [4] applies to our case as well.

2. Generalizations of nearly alternative rings. In this section we consider rings more general than nearly alternative rings. We shall call a power associative ring $R$ an $F$ ring if $R$ satisfies the following identities:

$$
\begin{gathered}
\left(w, x^{2}, z\right)=x \cdot(w, x, z) \\
([x, y], y, y)=0 .
\end{gathered}
$$

That an $F$ ring is a weaker concept than a nearly alternative ring is shown by an example due to Anderson [3] of a power associative algebra satisfying (2.1) and (2.2) which is not flexible; hence not noncommutative Jordan. We are able to prove, however, that a flexible $F$ ring is noncommutative Jordan.

LEMMA 2.1. In a flexible $F$ ring the following equations hold:

$$
\begin{gathered}
(x \cdot y, z, w)+(x, y, z \cdot w)=x \cdot(y, z, w)+y \cdot(x, z, w) \\
+z \cdot(x, y, w)+w \cdot(x, y, z) \\
{[x,(x, x, y)]=0} \\
\left(x^{2}, y, x\right)=x(x, x, y)-(x, x, x y) .
\end{gathered}
$$

Proof. Property (2.3) is proved in Lemma 1.2 using only (2.1) and flexibility. For property (2.4) we use the Teichmüller identity (1.10) twice to get $(x y, z, w)+(x, y, z w)-(x, y z, w)-x(y, z, w)-$ $(x, y, z) w=0$ and $(w z, y, x)+(w, z, y x)-(w, z y, x)-w(z, y, x)-$ $(w, z, y) x=0$. Add these equations to obtain by flexibility

$$
\begin{aligned}
(x, y,[z, w]) & -(w,[z, y], x)+([x, y], z, w)-[x,(y, z, w)] \\
& +[w,(x, y, w)]=0 .
\end{aligned}
$$

Let $z=x, y=x, w=y$ in (2.6). Then it follows that $(x, x,[x, y])-$ $(y,[x, x], x)+([x, x], x, y)-[x,(x, x, y)]+[y,(x, x, x)]=0, \quad$ and $[x,(x, x, y)]=0$.

To prove property (2.5), let $y=x, w=x, z=y$ in (2.3). Then $(x \cdot x, y, x)+(x, x, y \cdot x)=x \cdot(x, y, x)+x \cdot(x, y, x)+y \cdot(x, x, x)+x \cdot(x, x, y)$ becomes 


$$
2\left(x^{2}, y, x\right)+(x, x, y \cdot x)-x \cdot(x, x, y)=0 .
$$

But property $(2.4)$ implies $x \cdot(x, x, y)=2 x(x, x, y)$, and $(x, x,[x, y])=0$ implies $(x, x, x y)=(x, x, y x)$. Hence (2.7) becomes $2\left(x^{2}, y, x\right)+2(x, x, x y)-$ $2 x(x, x, y)=0$. Since $R$ is 2-torsion free, $\left(x^{2}, y, x\right)=x(x, x, y)-$ $(x, x, x y)$.

THEOREM 2.1. A flexible $F$ ring is a noncommutative Jordan ring.

Proof. Since $R$ is power associative $\left(x, x, x^{2}\right)=0$. Partially linearize $\left(x, x, x^{2}\right)=0$ to get

$$
(x, x, x y)+(x, x, y x)+\left(x, y, x^{2}\right)+\left(y, x, x^{2}\right)=0 .
$$

\section{This implies}

$$
2(x, x, x y)+\left(y, x, x^{2}\right)=\left(x^{2}, y, x\right) \text {. }
$$

Subtracting (2.5) from (2.9) gives $3(x, x, x y)-x(x, x, y)+\left(y, x, x^{2}\right)=$ 0 or

$$
\left(x^{2}, x, y\right)=3(x, x, x y)-x(x, x, y) \text {. }
$$

Now property (2.3) with $z=y=x, w=y$ gives $2\left(x^{2}, x, y\right)+2(x, x, x y)=$ $6 x(x, x, y)$ which becomes

$$
\left(x^{2}, x, y\right)=3 x(x, x, y)-(x, x, x y) .
$$

Subtracting (2.11) from (2.10) gives $4 x(x, x, y)-4(x, x, x y)=0$ or

$$
x(x, x, y)=(x, x, x y) .
$$

Substitute (2.12) in (2.5) to get $\left(x^{2}, y, x\right)=0$. The theorem is thus proved.

We next consider flexible rings which satisfy the identity

$$
\left(w, x^{2}, z\right)=x \cdot(w, x, z)+(x, x,[w, z]) .
$$

THEOREM 2.2. If $R$ is a simple flexible ring which satisfies identity (2.13) and $e \neq 1$ is an idempotent of $R$ such that $(e, e, R)=$ 0 then $R$ is alternative.

Proof. Since $(e, e, R)=(R, e, e)=(e, R, e)=0, R$ has Peirce decomposition into the direct sum $R=R_{1}+R_{10}+R_{01}+R_{0}$ where $R_{i}=$ $\{x \in R \mid e x=x e=i x\}$ for $i=0,1$, and $R_{i j}=\{x \in R \mid e x=i x, x e=j x\}$ for $i, j=0,1, i \neq j$. We first determine the multiplication table of the decomposition as 


\begin{tabular}{c|cccc}
\multicolumn{1}{c}{} & \multicolumn{1}{c}{$R_{1}$} & $R_{10}$ & $R_{01}$ & $R_{0}$ \\
\cline { 2 - 5 }$R_{1}$ & $R_{1}$ & $R_{10}$ & 0 & 0 \\
$R_{10}$ & 0 & $R_{01}$ & $R_{1}$ & $R_{10}$ \\
$R_{01}$ & $R_{01}$ & $R_{0}$ & $R_{10}$ & 0 \\
$R_{0}$ & 0 & 0 & $R_{01}$ & $R_{0}$
\end{tabular}

Linearize identity (2.13) to get

$$
\begin{aligned}
(w, x \cdot y, z)= & x \cdot(w, y, z)+y \cdot(w, x, z)+(x, y,[w, z]) \\
& +(y, x,[w, z]) .
\end{aligned}
$$

Flexibility clearly implies $R_{10} R_{1}=R_{01} R_{0}=R_{0} R_{10}=0$ and $R_{i j} R_{j} \subseteq R_{i j}$, $R_{i} R_{i j} \cong R_{i j}$ for $i, j=0,1, i \neq j$. For $x_{1}, y_{1} \in R_{1},\left(x_{1}, y_{1}, e\right)=-\left(e, y_{1}, x_{1}\right)$ implies $\left(x_{1} y_{1}\right)_{10}=0$ and $\left(y_{1} x_{1}\right)_{01}=0$ or $R_{1} R_{1} \subseteq R_{0}+R_{1}$. But $\left(x_{1}, y_{1} \cdot e, e\right)=$ $y_{1} \cdot\left(x_{1}, e, e\right)+e \cdot\left(x_{1}, y_{1}, e\right)+\left(y_{1}, e,\left[x_{1}, e\right]\right)+\left(e, y_{1},\left[x_{1}, e\right]\right)$ implies $2\left(x_{1}, y_{1}, e\right)=$ $e \cdot\left(x_{1}, y_{1}, e\right)$ or $2\left(x_{1} y_{1}\right) e-2 x_{1} y_{1}=e \cdot\left[\left(x_{1} y_{1}\right) e-x_{1} y_{1}\right]$. Hence $\left(x_{1} y_{1}\right)_{0}=0$ and $R_{1} R_{1} \subseteq R_{1}$. In a similar manner $R_{0} R_{0} \subseteq R_{0}$. Again by flexibility, $\left(x_{1}, y_{0}, e\right)=-\left(e, y_{0}, x_{1}\right)$ and $x_{1} y_{0} \in R_{1}+R_{0}$. Also $\left(x_{1}, e, y_{0}\right)=-\left(y_{0}, e, x_{1}\right)$ implies $x_{1} y_{0}=y_{0} x_{1}$. Applying (2.14) yields $\left(x_{1}, e \cdot y_{0}, e\right)=e \cdot\left(x_{1}, y_{0}, e\right)+$ $y_{0} \cdot\left(x_{1}, e, e\right)+\left(e, y_{0},\left[x_{1}, e\right]\right)+\left(y_{0}, e,\left[x_{1}, e\right]\right)$ or $e \cdot\left(x_{1}, y_{0}, e\right)=0$. This gives $\left(x_{1} y_{0}\right)_{1}=0$. Again by $(2.14),\left(y_{0}, e \cdot x_{1}, e\right)=e \cdot\left(y_{0}, x_{1}, e\right)+x_{1} \cdot\left(y_{0}, e, e\right)+$ $\left(e, x_{1},\left[y_{0}, e\right]\right)+\left(x_{1}, e,\left[y_{0}, e\right]\right)$ which implies $2\left(y_{0}, x_{1}, e\right)=e \cdot\left(y_{0}, x_{1}, e\right)$ or $2\left(y_{0} x_{1}\right) e-2 y_{0} x_{1}=e \cdot\left[\left(y_{0} x_{1}\right) e-y_{0} x_{1}\right]$. This gives $\left(y_{0} x_{1}\right)_{0}=\left(x_{1} y_{0}\right)_{0}=0$ and $R_{1} R_{0}=R_{0} R_{1}=0$. Therefore $R_{0}, R_{1}$ are orthogonal subrings. Now by identity (2.14), $\left(e, x_{10} \cdot e, y_{10}\right)=x_{10} \cdot\left(e, e, y_{10}\right)+e \cdot\left(e, x_{10}, y_{10}\right)+\left(x_{10}, e,\left[e, y_{10}\right]\right)+$ $\left(e, x_{10},\left[e, y_{10}\right]\right)$ or $x_{10} y_{10}-e\left(x_{10} y_{10}\right)=e \cdot\left[x_{10} y_{10}-e\left(x_{10} y_{10}\right)\right]-x_{10} y_{10}+x_{10} y_{10}-$ $e\left(x_{10} y_{10}\right)$. This becomes $x_{10} y_{10}=e \cdot\left[x_{10} y_{10}-e\left(x_{10} y_{10}\right)\right]$ and $x_{10} y_{10} \in R_{01}$. We have $R_{10} R_{10} \subseteq R_{01}$. Similarly $R_{01} R_{01} \subseteq R_{10}$. In the case $R_{10} R_{01}$, apply (2.14) to obtain $\left(e, x_{10} \cdot e, y_{01}\right)=x_{10} \cdot\left(e, e, y_{01}\right)+e \cdot\left(e, x_{10}, y_{01}\right)+\left(x_{10}, e,\left[e, y_{01}\right]\right)+$ $\left(e, x_{10},\left[e, y_{01}\right]\right)$ or $\left(x_{10} y_{01}\right)-e\left(x_{10} y_{01}\right)=e \cdot\left[x_{10} y_{01}-e\left(x_{10} y_{01}\right)\right]-x_{10} y_{01}+e\left(x_{10} y_{01}\right)$. This becomes $2\left(x_{10} y_{01}\right)-2 e\left(x_{10} y_{01}\right)=e \cdot\left[x_{10} y_{01}-e\left(x_{10} y_{01}\right)\right]$, and $x_{10} y_{01} \in R_{1}+$ $R_{10}$. Apply (2.14) once more to obtain $\left(e, y_{01} \cdot e, x_{10}\right)=y_{01} \cdot\left(e, e, x_{10}\right)+$ $e \cdot\left(e, y_{01}, x_{10}\right)+\left(y_{01}, e,\left[e, x_{10}\right]\right)+\left(e, y_{01},\left[e, x_{10}\right]\right)$ which becomes $e \cdot\left(e, y_{01}, x_{10}\right)=$ 0 since $\left(y_{01}, e, x_{10}\right)=-\left(x_{10}, e, y_{01}\right)=0$. Thus $e \cdot\left(x_{10}, y_{01}, e\right)=e \cdot\left[\left(x_{10} y_{01}\right) e-x_{10} y_{01}\right]=$ 0 and $\left(x_{10} y_{01}\right)_{10}=0$. It follows that $R_{10} R_{01} \subseteq R_{1}$ and $R_{01} R_{10} \subseteq R_{0}$. In a similar manner using flexibility and identity (2.14) the multiplication chart is verified.

That $B=R_{10}+R_{01}+R_{10} R_{01}+R_{01} R_{10}$ is an ideal of $R$ follows from flexiblity and the multiplicative properties of the subrings. If $B=$ $0, R_{10}=R_{01}=0$ and $R=R_{0}+R_{1}$, a contradiction. $B=R$ implies $R_{10} R_{01}=R_{1}, R_{01} R_{10}=R_{0}$, and $\left(R_{1}, R_{1}, R_{1}\right)=0$ since $\left(x_{1}, y_{1}, z_{1}\right)=\left(x_{1}, y_{10} \cdot y_{01}, z_{1}\right)=$ $y_{10} \cdot\left(x_{1}, y_{01}, z_{1}\right)+y_{01} \cdot\left(x_{1}, y_{10}, z_{1}\right)+\left(y_{10}, y_{01},\left[x_{1}, z_{1}\right]\right)+\left(y_{01}, y_{10},\left[x_{1}, z_{1}\right]\right)$ or $\left(x_{1}, y_{1}, z_{1}\right)=-\left(\left[x_{1}, z_{1}\right], y_{01}, y_{10}\right)=0$. Similarly $\left(R_{0}, R_{0}, R_{0}\right)=0$. 
For alternativity, we first consider $\left(R_{1}, R_{10}, R_{10}\right)$. We observe that for $x_{10} \in R_{10},\left(x_{10}, x_{10}, e\right)=-\left(e, x_{10}, x_{10}\right)$ implies $x_{10}^{2}=0$ and $\left(x_{10}+y_{10}\right)^{2}=$ 0 implies $x_{10} y_{10}=-y_{10} x_{10}$. Therefore $\left(x_{1}, y_{10}, z_{10}\right)=-\left(z_{10}, y_{10}, x_{1}\right)$ implies $\left(x_{1} y_{10}\right) z_{10}=-\left(z_{10} y_{10}\right) x_{1}$ and $\left(x_{1}, z_{10}, y_{10}\right)=\left(x_{1} z_{10}\right) y_{10}=-\left(y_{10} z_{10}\right) x_{1}=\left(z_{10} y_{10}\right) x_{1}=$ $\left(x_{1}, y_{10}, z_{10}\right)$. Also $\left(z_{10}, x_{1}, y_{10}\right)=-z_{10}\left(x_{1} y_{10}\right)=\left(x_{1} y_{10}\right) z_{10}=\left(x_{1}, y_{10}, z_{10}\right)=-$ $\left(z_{10}, y_{10}, x_{1}\right)$. Therefore we have $\left(x_{1}, y_{10}, z_{10}\right)=-\left(z_{10}, y_{10}, x_{1}\right)=\left(z_{10}, x_{1}, y_{10}\right)=$ $-\left(y_{10}, x_{1}, z_{10}\right)=\left(y_{10}, z_{10}, x_{1}\right)=-\left(x_{1}, z_{10}, y_{10}\right)$, and $\left(R_{1}, R_{10}, R_{10}\right)$ alternates. Similarly $\left(R_{1}, R_{01}, R_{01}\right)$ alternates.

That all other associators with at least one $R_{1}$ in any position alternate follows from the chart, flexibility, and $\left(R_{1}, R_{1}, R_{1}\right)=0$. Likewise we can see that all associators involving at least one $R_{0}$ in any position alternate.

It remains to verify $\left(R_{i j}, R_{i j}, R_{i j}\right)$ and $\left(R_{j i}, R_{i j}, R_{i j}\right)$ with $i, j=$ $0,1, i \neq j$ alternate. Letting $x_{10}, y_{10}, z_{10} \in R_{10}$ and applying (2.14) we obtain $\left(x_{10}, y_{10} \cdot e, z_{10}\right)=y_{10} \cdot\left(x_{10}, e, z_{10}\right)+e \cdot\left(x_{10}, y_{10}, z_{10}\right)+\left(y_{10}, e,\left[x_{10}, z_{10}\right]\right)+$ $\left(e, y_{10},\left[x_{10}, z_{10}\right]\right)$ which becomes $\left(x_{10} y_{10}\right) z_{10}-x_{10}\left(y_{10} z_{10}\right)=y_{10} \cdot\left(-x_{10} z_{10}\right)+$ $e \cdot\left[\left(x_{10} y_{10}\right) z_{10}-x_{10}\left(y_{10} z_{10}\right)\right]+2 y_{10}\left(x_{10} z_{10}\right)-2 e\left[y_{10}\left(x_{10} z_{10}\right)\right]$ or $\left(x_{10} y_{10}\right) z_{10}-x_{10}\left(y_{10} z_{10}\right)=$ $2 x_{10}\left(y_{10} z_{10}\right)-y_{10} \cdot\left(x_{10} z_{10}\right)$. Since $R$ is a direct sum, $\left(x_{10} y_{10}\right) z_{10}=-\left(x_{10} z_{10}\right) y_{10}$ and $x_{10}\left(y_{10} z_{10}\right)=-y_{10}\left(x_{10} z_{10}\right)$. This implies $\left(x_{10}, z_{10}, y_{10}\right)=\left(x_{10} z_{10}\right) y_{10}$ $x_{10}\left(z_{10} y_{10}\right)=-\left(z_{10} x_{10}\right) y_{10}+z_{10}\left(x_{10} y_{10}\right)=-\left(z_{10}, x_{10}, y_{10}\right)$. Also $\left(y_{10}, x_{10}, z_{10}\right)=$ $\left(y_{10} x_{10}\right) z_{10}-y_{10}\left(x_{10} z_{10}\right)=-\left(x_{10} y_{10}\right) z_{10}+x_{10}\left(y_{10} z_{10}\right)=-\left(x_{10}, y_{10}, z_{10}\right)$. We have $\left(x_{10}, y_{10}, z_{10}\right)=-\left(z_{10}, y_{10}, x_{10}\right)=-\left(y_{10}, x_{10}, z_{10}\right)=\left(z_{10}, x_{10}, y_{10}\right)=-\left(x_{10}, z_{10}, y_{10}\right)=$ $\left(y_{10}, z_{10}, x_{10}\right)$. This proves $\left(R_{10}, R_{10}, R_{10}\right)$ and similarly $\left(R_{01}, R_{01}, R_{01}\right)$ alternate. Lastly, let $x_{10}, y_{10} \in R_{10}, z_{01} \in R_{01}$. It follows that $\left(z_{01}, y_{10}, x_{10}\right)=$ $-\left(x_{10}, y_{10}, z_{01}\right)=-\left(x_{10} y_{10}\right) z_{01}=\left(y_{10} x_{10}\right) z_{01}=\left(y_{10}, x_{10}, z_{01}\right)=-\left(z_{01}, x_{10}, y_{10}\right)$. Also by (2.14), $\left(e, x_{10} \cdot z_{01}, y_{10}\right)=x_{10} \cdot\left(e, z_{01}, y_{10}\right)+z_{01} \cdot\left(e, x_{10}, y_{10}\right)+\left(x_{10}, z_{01}\right.$, $\left.\left[e, y_{10}\right]\right)+\left(z_{01}, x_{10},\left[e, y_{10}\right]\right)$ which becomes $0=z_{01} \cdot\left(x_{10} y_{10}\right)+\left(x_{10}, z_{01}, y_{10}\right)+$ $\left(z_{01}, x_{10}, y_{10}\right)$. But $z_{01} \cdot\left(x_{10} y_{10}\right)=0$ since $x_{10} y_{10} \in R_{01}$ and $\left(x_{10}, z_{01}, y_{10}\right)=$ $-\left(z_{01}, x_{10}, y_{10}\right)$. We therefore have $\left(x_{10}, y_{10}, z_{01}\right)=-\left(z_{01}, y_{10}, x_{10}\right)=$ $\left(y_{10}, z_{01}, x_{10}\right)=-\left(x_{10}, z_{01}, y_{10}\right)=\left(z_{01}, x_{10}, y_{10}\right)=-\left(y_{10}, x_{10}, z_{01}\right)$. This shows that $\left(R_{01}, R_{10}, R_{10}\right)$ and by a reversal of subscripts that $\left(R_{10}, R_{01}, R_{01}\right)$ alternate. The theorem is proved.

In the case of a finite dimensional algebra $A$ we can prove the following:

Theorem. If $A$ is a simple flexible finite dimensional power associative algebra over an algebraically closed field $F$ of characteristic $\neq 2,3$ which satisfies $\left(w, x^{2}, z\right)=x \cdot(w, x, z)+(x, x,[w, z])$ and $e \neq 1$ is an idempotent of $A$ then $A$ is noncommutative Jordan.

Proof. Oehmke $[9,10]$ has shown that a simple, flexible, stable, finite dimensional power associative algebra over an algebraically closed field of characteristic $\neq 2,3$ is a noncommutative Jordan algebra. We show $A$ is stable, i.e., $A_{i} A_{1 / 2} \subseteq A_{1 / 2}$ and $A_{1 / 2} A_{i} \subseteq A_{1 / 2}$ for $i=0,1$. 
Since $A$ is power associative, $A=A_{1}+A_{1 / 2}+A_{0}$ where $A_{i}=$ $\{x \in A \mid e x+x e=i x\}$. Also $A_{1} A_{0}=A_{0} A_{1}=0, A_{i} A_{i} \cong A_{i}$ for $i=0,1$, $A_{1 / 2} A_{1 / 2} \subseteq A_{1}+A_{0}$, and $A_{i} A_{1 / 2} \subseteq A_{1 / 2}+A_{1-i}, A_{1 / 2} A_{i} \subseteq A_{1 / 2}+A_{1-i}$ for $i=0,1$.

By flexibility $\left(e, x_{1 / 2}, e\right)=0$ implies $e\left(x_{1 / 2} e\right)=\left(e x_{1 / 2}\right) e$, and $x_{1 / 2}=$ $e x_{1 / 2}+x_{1 / 2} e$ implies $e x_{1 / 2}=e\left(e x_{1 / 2}\right)+e\left(x_{1 / 2} e\right)$ or $e x_{1 / 2}=e\left(e x_{1 / 2}\right)+\left(e x_{1 / 2}\right) e$. Hence $e x_{1 / 2} \in A_{1 / 2}$ and $x_{1 / 2} e \in A_{1 / 2}$.

Next we consider $A_{1} A_{1 / 2}$. By identity (2.14), for $x \in A_{1}, y \in A_{1 / 2}$, $(x, \dot{y} \cdot e, e)=y \cdot(x, e, e)+e \cdot(x, y, e)+(y, e,[x, e])+(e, y,[x, e])$ and $(x, y, e)=e \cdot(x, y, e)$, i.e., $(x, y, e) \in A_{1 / 2}$. We also have $(x, e \cdot e, y)=$ $2(x, e, y)=2 e \cdot(x, e, y)+2(e, e,[x, y])$. Therefore $(x, e, y)_{1}=(x, e, y)_{0}=0$ and $(x, e, y) \in A_{1 / 2} \cdot \quad$ Again by (2.14), $(e, x \cdot e, y)=x \cdot(e, e, y)+e \cdot(e, x, y)+$ $(x, e,[e, y])+(e, x,[e, y])$. Since $A$ is a direct sum and $(x, e,[e, y]) \in$ $A_{1 / 2}$, it follows that

$$
2(e, x, y)_{0}=[x \cdot(e, e, y)]_{0}+(e, x,[e, y])_{0} .
$$

Apply the Teichmüller identity (1.10) to get

$$
(e, x, e y)=-(e x, e, y)+(e, x e, y)+e(x, e, y)+(e, x, e) y
$$

and

$$
(e, x, y e)=-(e x, y, e)+(e, x y, e)+e(x, y, e)+(e, x, y) e .
$$

But the $A_{0}$ components of (2.16) give $(e, x, e y)_{0}=(e, x, y)_{0}$ and those of (2.17) give $(e, x, y e)_{0}=[(e, x, y) e]_{0}=0$. Substituting these results in (2.15) yields $2(e, x, y)_{0}=[x \cdot(e, e, y)]_{0}+(e, x, e y)_{0}-(e, x, y e)_{0}$. This becomes

$$
(e, x, y)_{0}=[x \cdot(e, e, y)]_{0} \text {. }
$$

Now consider $[x \cdot(e, e, y)]_{0}$. As in $[4], x \cdot(e, e, y)=x y-(x, e, y)-$ $x(e y)+(x, e, e y)+e(y x)+(e, y, x)-e[(e y) x]-(e, e y, x)$. All terms on the right side except the first and third are in $A_{1 / 2}$. Therefore

$$
[x \cdot(e, e, y)]_{0}=(x y)_{0}-[x(e y)]_{0} .
$$

Substitute (2.19) into (2.18) to get

$$
(e, x, y)_{0}=(x y)_{0}-[x(e y)]_{0} .
$$

Identity $(2.20)$, expanded becomes $(x y)_{0}-[e(x y)]_{0}=(x y)_{0}-[x(e y)]_{0}$. Since $[e(x y)]_{0}=0$ it follows that $[x(e y)]_{0}=0$, and since $(x, e, y) \in A_{1 / 2}$, it follows that $(x y)_{0}-[x(e y)]_{0}=0$. We have therefore $(x y)_{0}=0$ or $A_{1} A_{1 / 2} \subseteq A_{1 / 2}$. Identity $(2.20)$ becomes $(e, x, y)_{0}=0$ and by flexibility $(y, x, e)_{0}=0$. Thus $[(y x) e]_{0}-(y x)_{0}=0$. This gives $A_{1 / 2} A_{1} \subseteq A_{1 / 2}$. In a similar manner $A_{0} A_{1 / 2} \subseteq A_{1 / 2}, A_{1 / 2} A_{0} \subseteq A_{1 / 2}$ and $A$ is stable. The theorem is therefore proved. 


\section{REFERENCES}

1. A. A. Albert, Power associative rings, Trans. Amer. Math. Soc., 64 (1948), 552-593.

2. _- Almost alternative algebras, Portugal. Math., 8 (1949), 23-36.

3. C. T. Anderson, A note on derivations of commutative algebras, Proc. Amer. Math. Soc., 17 (1966), 1199-1202.

4. R. Block, $A$ unification of the theories of Jordan and alternative algebras, Amer. J. Math., 94 (1972), 389-412.

5. F. Florey, A generalization of noncommutative Jordan algebras, Algebra, 23 (1972), 502-518.

6. E. Kleinfeld, and L. Kokoris, Flexible algebras of degree one, Proc. Amer. Math. Soc., 13 (1962), 891-893.

7. L. Kokoris, Nodal nocommutaitve Jordan algebras, Canad. Math., 12 (1960), 488-492.

8. K. McCrimmon, Structure and representation of noncommutative Jordan algebras, Trans. Amer. Math. Soc., 121 (1966), 187-199.

9. R. H. Oehmke, On flexible algebras, Ann. of Math., 68 (1958), 221-230.

10. On flexible power associative algebras of degree two, Proc. Amer. Math. Soc., 12 (1961), 151-158.

11. R. D. Schaefer, On noncommutative Jordan algebras, Proc. Amer. Math. Soc., 9 (1958), 110-117.

12. - Introduction to Nonassociative Algebras, Academic Press, New York and London, 1966.

13. P. Shestakov, Certain classes of noncommutative Jordan rings, Algebra Logic, 10 (1971), 407-488.

Received April 1, 1976 and in revised form April 26, 1977. This paper includes portions of the author's $\mathrm{Ph}$. D. thesis completed under the direction of Professor Michael Rich at Temple University.

VILLANOVA UNIVERSITY

VILLANOVA, PA 19085 



\section{PACIFIC JOURNAL OF MATHEMATICS}

EDITORS

RICHARD ARENS (Managing Editor)

University of California

Los Angeles, California 90024

C. W. Curtis

University of Oregon

Eugene, OR 97403

C. C. MOORE

J. DUGUNDJI

Department of Mathematics University of Southern Californa Los Angeles, California 90007

R. Finn AND J. Milgram Stanford University Stanford, California 94305

University of California

Berkeley, CA 94720

\section{ASSOCIATE EDITORS}

E. F. BeCKENBACH

B. H. NeUMANN

F. WOLF

K. YosHida

\section{SUPPORTING INSTITUTIONS}

UNIVERSITY OF BRITISH COLUMBIA UNIVERSITY OF SOUTHERN CALIFORNIA CALIFORNIA INSTITUTE OF TECHNOLOGY UNIVERSITY OF CALIFORNIA MONTANA STATE UNIVERSITY UNIVERSITY OF NEVADA, RENO STANFORD UNIVERSITY UNIVERSITY OF TOKYO UNIVERSITY OF UTAH NEW MEXICO STATE UNIVERSITY WASHINGTON STATE UNIVERSITY OREGON STATE UNIVERSITY UNIVERSITY OF WASHINGTON UNIVERSITY OF OREGON OSAKA UNIVERSITY 


\section{Pacific Journal of Mathematics \\ Vol. 73, No. 1 \\ March, 1977}

Thomas Robert Berger, Hall-Higman type theorems. $V \ldots \ldots \ldots \ldots \ldots \ldots \ldots$

Frank Peter Anthony Cass and Billy E. Rhoades, Mercerian theorems via



Morris Leroy Eaton and Michael David Perlman, Generating $\mathrm{O}(n)$ with

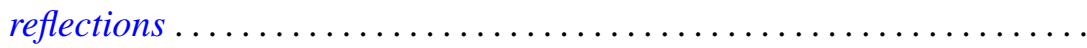

Frank John Forelli, Jr., A necessary condition on the extreme points of a

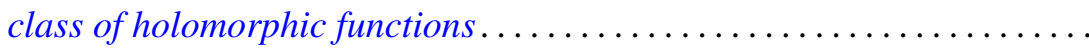

Melvin F. Janowitz, Complemented congruences on complemented

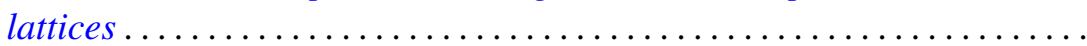

Maria M. Klawe, Semidirect product of semigroups in relation to amenability, cancellation properties, and strong $F \phi$ lner conditions....

Theodore Willis Laetsch, Normal cones, barrier cones, and the "spherical image" of convex surfaces in locally convex spaces ................

Chao-Chu Liang, Involutions fixing codimension two knots.............

Joyce Longman, On generalizations of alternative algebras .............

Giancarlo Mauceri, Square integrable representations and the Fourier

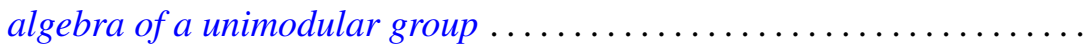

J. Marshall Osborn, Lie algebras with descending chain condition...

John Robert Quine, Jr., Tangent winding numbers and branched

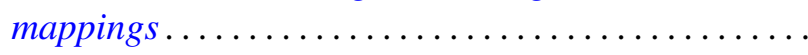

Louis Jackson Ratliff, Jr. and David Eugene Rush, Notes on ideal covers and associated primes .

H. B. Reiter and N. Stavrakas, On the compactness of the hyperspace of faces.

Walter Roth, A general Rudin-Carlson theorem in Banach-spaces ..

Mark Andrew Smith, Products of Banach spaces that are uniformly rotund in every direction.

Roger R. Smith, The R-Borel structure on a Choquet simplex ...

Gerald Stoller, The convergence-preserving rearrangements of real infinite series. ...

Graham H. Toomer, Generalized homotopy excision theorems modulo a Serre class of nilpotent groups...

Norris Freeman Weaver, Dehn's construction and the Poincaré conjecture....

Steven Howard Weintraub, Topological realization of equivariant intersection forms... 